\title{
O Comportamento de Crianças Durante Atendimento Odontológico ${ }^{1}$
}

\author{
Rosana de Fátima Possobon ${ }^{2}$ \\ Faculdade de Odontologia de Piracicaba - UNICAMP \\ Antonio Bento Alves de Moraes \\ Faculdade de Odontologia de Piracicaba - UNICAMP \\ Áderson Luiz Costa Junior \\ Universidade de Brasília \\ Gláucia Maria Bovi Ambrosano \\ Faculdade de Odontologia de Piracicaba - UNICAMP
}

\begin{abstract}
RESUMO - Este trabalho avaliou o comportamento de 6 crianças com história de não - colaboração durante tratamento odontológico, que passaram por 5 sessões odontológicas, nas quais empregou-se o placebo ou o diazepam de maneira duplo - cega, além de estratégias psicológicas de manejo do comportamento (distração, explicação, reforçamento e estabelecimento de regras). As sessões foram filmadas em vídeo - tape, com marcas sonoras a cada 15 segundos, indicativas dos momentos em que os comportamentos emitidos pelos participantes (choro, movimentos de corpo e/ou cabeça, fuga e esquiva) e as estratégias de manejo do comportamento seriam registrados. Os resultados mostraram que o medicamento na dose utilizada foi eficaz para controlar os comportamentos de 1 participante, sendo que os demais não permitiram a realização do tratamento e exibiram aumento crescente da resistência ao tratamento. Parece necessário que a criança seja auxiliada a enfrentar a situação de tratamento nas sessões iniciais, impedindo o aumento da resistência.
\end{abstract}

Palavras-chave: crianças não-colaboradoras; comportamento; controle farmacológico; estratégias de manejo; odontologia infantil.

\section{Child Behavior During Dental Treatment}

\begin{abstract}
This research intended to investigate the use of diazepam in conjunction with behavioral strategies to manage uncooperative behavior of child dental patients. The 6 participants received dental treatment during 9 sessions. Using a double-blind design, children received placebo or diazepam and at the same time were submitted to behavior management produces (distraction, explanation, reinforcement and set rule and limits). All sessions were recorded in video-tapes biped in 15 seconds intervals, in which observers recorded child's (crying, body and/or head movements, escape and avoidance) and dentist's behavior. The results indicated that diazepam, considering the used dose, was only effective with one subject. The other participants didn't permit the treatment and showed an increase in their resistance. The behavioral preparation strategies for dental treatment should have been more precisely planned in order to help the child to face the real dental treatment conditions mainly in the first sessions avoiding to reinforce inappropriate behaviors.
\end{abstract}

Key words: uncooperative children; behavior control; pharmacological control; dental child.

O tratamento odontológico, em muitas circunstâncias, é um grande desafio para o paciente e para o profissional, devido às condições aversivas presentes (César, 1988). O medo leva o indivíduo a cancelar ou adiar suas consultas odontológicas, agravando a sua condição de saúde bucal (Costa \& Moraes, 1994).

Ao lidar com o comportamento de pacientes na faixa etária pré - escolar fica claro que, além das dificuldades em colaborar com o tratamento, inerentes à idade, tais como permanecer imóvel por alguns minutos com a boca aberta, o medo específico do tratamento pode impedir a atuação adequada do profissional. Histórias de situações odontológicas

1 Artigo derivado da Dissertação de Mestrado: Uso combinado de estratégias comportamentais e farmacológicas no manejo da criança não - colaboradora durante o atendimento odontológico. Agradecimentos à psicóloga Fabíola Cristina Trivelatto e ao Técnico em Imagem e Som: Paulo Roberto Rizzo do Amaral.

2 Rua Prudente de Moraes, $n^{\circ}$ 1835. Piracicaba - SP. CEP 13416-720. E.mail: possobon@fop.unicamp.br aversivas representam um conjunto significativo de variáveis predisponentes para respostas de medo em crianças. Fazem parte dessas histórias a qualidade da interação dentista - criança e sensações dolorosas muitas vezes inevitáveis (Moraes, 1999). Assim, a experiência clínica demonstra que é mais fácil lidar com os comportamentos de uma criança que não tem experiência odontológica do que manejar os comportamentos daquelas que já tiveram experiências desagradáveis no dentista (Piedalue \& Milnes, 1994).

Embora medo e comportamentos de não - colaboração possam representar uma mesma manifestação psicológica, nem todo comportamento de não - colaboração representa o medo e nem toda criança temerosa é não - colaboradora (Ingersoll, 1982). Pinkham (1993) afirma que o medo (como um sentimento) pode ser um fator predisponente para comportamentos de esquiva, mas que estes comportamentos também podem ocorrer com crianças já familiarizadas com o tratamento. Isto equivale dizer que a presença de estímulos aversivos aumenta a probabilidade de ocorrência de respostas de esquiva. O padrão e a freqüência de respostas de esquiva 
são parâmetros necessários para o reconhecimento de uma criança temerosa.

A criança não - colaboradora pode ser considerada um paciente especial, por apresentar dificuldades em ajustar-se às exigências do tratamento odontológico convencional, necessitando de alterações na rotina do atendimento, por meio da introdução de estratégias de manejo, que não são utilizadas regularmente pelos cirurgiões - dentistas. Em Odontologia, o termo manejo significa uma interação contínua com a criança, direcionada pela comunicação e educação em um esforço para reduzir ou eliminar o medo e a ansiedade e promover um melhor entendimento, não apenas da necessidade de uma boa saúde dentária, como também do processo pela qual é conseguida (American Academy of Pediatric Dentistry, 1996-1997).

A despeito de todo o cuidado na escolha e implementação da estratégia de manejo mais adequada a cada situação, algumas crianças mostram-se refratárias ou mais resistentes.

Allen, Loiben, Allen e Stanley (1992) utilizaram a contingência de fuga para manejar os comportamentos de quatro crianças que apresentavam níveis baixos de colaboração. Esta estratégia consiste em interromper o tratamento por alguns segundos para que a criança descanse, após manifestações de colaboração. As crianças apresentaram uma redução nos comportamentos não - colaboradores durante as sessões em que houve o uso da fuga contingente. Entretanto, os autores sugerem que, se o nível de não - colaboração das crianças fosse maior, esta estratégia teria sido insuficiente para manejá-las.

Ingersoll (1982), ao estudar pacientes odontopediátricos considerados "incontroláveis", ou seja, com alto nível de comportamentos de não - colaboração, afirma que, para estas crianças, estratégias psicológicas como a distração, não promovem mudanças comportamentais que permitam a adequada atuação do dentista. Nível alto de não - colaboração é um termo utilizado para designar uma forte recusa da criança em aceitar as regras do tratamento ou uma medida da duração das manifestações de não - colaboração que excede a 90\% do tempo de uma sessão de atendimento odontológico. A autora cita que, a mesma estratégia, utilizada com crianças com níveis mais baixos de não - colaboração, apresenta resultados mais favoráveis. Isto parece ocorrer porque a criança considerada "incontrolável" não entra em contato com os comportamentos do dentista (o que ele diz ou faz para acalmá-la), mas permanece sob controle de outros estímulos relevantes para a manutenção da resistência ou rebeldia.

Além do nível de não - colaboração inicial, parece haver uma tendência a aumentar a resistência das crianças ao tratamento ao longo das sessões, quando o dentista introduz gradualmente no tratamento os procedimentos considerados mais aversivos, seguindo, em termos de procedimento, uma orientação derivada da dessensibilização sistemática (Wolpe, 1971). Em um estudo com dois pacientes especiais (um não - colaborador e um portador de deficiência mental), César (1988) encontrou níveis crescentes de resistência ao longo das sessões de atendimento odontológico. Vale a pena ressaltar que, neste caso, as crianças foram atendidas por alunos de graduação pouco experientes para as tarefas clínicas e de manejo do comportamento. Achados semelhantes foram obtidos por Stark, Allen, Hurst, Nash,
Rigney e Stokes (1989), que testaram a eficácia da técnica de distração, durante o atendimento odontológico de quatro crianças não - colaboradoras. Os resultados mostraram que, após as visitas sucessivas, houve um crescente aumento nos níveis de não - colaboração. Isto torna clara a necessidade de investigar as relações de contingência, explícitas ou não, da situação de atendimento odontológico e, como decorrência, propor estratégias para diminuir a ansiedade e promover a manutenção dos comportamentos colaboradores até o término do tratamento.

Nestes casos, em que o uso exclusivo do controle comportamental não permite uma adequada atuação profissional, o emprego de substâncias farmacológicas, tais como os ansiolíticos (exemplo: diazepam), pode auxiliar diminuindo a ansiedade da criança e permitindo a realização do seu tratamento, uma vez que a ansiedade pode ser compreendida como um componente importante das reações de não - colaboração (Moraes \& Pessotti, 1985).

A utilização de sedação leve em pacientes odontopediátricos parece reduzir o tempo operatório de cada sessão, proporcionar um menor grau de agitação e choro e aumentar a sonolência e a cooperação (Album, 1961; Badalaty, Houpt, Koenigsberg, Mawell e Desjardins, 1990; Sams, Cook, Jackson e Roebuck, 1993). Entretanto, alguns autores, tais como Yanase, Braham, Fukuta \& Kurosu (1996) e Healy \& Hamilton (1971), acreditam que, dependendo do grau de não - colaboração da criança, o medicamento pode ser pouco eficaz, necessitando do emprego concomitante de outras estratégias.

O objetivo deste estudo foi verificar o nível de resistência ao tratamento apresentado a cada sessão, comparando sessões em que a criança recebeu uma medicação ansiolítica com sessões em que recebeu um placebo.

\section{Método}

Este trabalho avaliou o comportamento de 6 crianças na faixa etária de 4 a 5 anos completos, com história de não - colaboração que impediu a realização de tratamentos odontológicos anteriores. Estes participantes passaram por 5 sessões de atendimento odontológico, realizadas no Laboratório de Psicologia Aplicada (LPA), uma unidade do Centro de Pesquisa e Atendimento Odontológico para Pacientes Especiais (Cepae) da Faculdade de Odontologia de Piracicaba, Universidade Estadual de Campinas - São Paulo. Antes da escolha dos participantes, o projeto deste estudo foi avaliado e aprovado pelo Comitê de Ética em Pesquisa desta Faculdade. Além disto, os responsáveis pelos participantes autorizaram a realização do tratamento, assinando um termo de "Informação e Consentimento Livre e Esclarecido".

Todas as sessões foram gravadas em vídeo - tape (VT), com sons de "bip" a cada 15 segundos, que marcavam os momentos em que, posteriormente, foram feitos os registros dos comportamentos observados.

Na primeira sessão não foi administrado nenhum medicamento. A partir da segunda sessão, os participantes receberam, de maneira duplo - cego, o ansiolítico diazepam (0,3 mg/kg, p.o.) ou o placebo (Quadro 1). Estas substâncias eram administradas nas dependências do Cepae, 60 minutos antes do início da sessão. 
Quadro 1: Esquema representativo do delineamento experimental utilizado neste trabalho. A droga A representava o placebo e a droga $\mathrm{B}$ continha o princípio ativo do diazepam (com identificação efetuada após a coleta dos dados).

\begin{tabular}{|c|c|c|}
\hline Sessão & Grupo A & Grupo B \\
\hline $\mathbf{1}^{\mathrm{a}}$ & Sem droga & Sem droga \\
\hline $2^{a}$ & Droga A & Droga B \\
\hline $3^{\mathrm{a}}$ & Droga A & Droga B \\
\hline $4^{a}$ & Droga B & Droga A \\
\hline $5^{a}$ & Droga B & Droga A \\
\hline
\end{tabular}

Na primeira sessão, a criança e seu acompanhante eram encaminhados ao consultório e, por meio de atividade lúdica (brincadeiras utilizando fantoches e instrumentos odontológicos) a dentista explicava e demonstrava os procedimentos que seriam realizados e como deveria ser o comportamento da criança durante o tratamento. A dentista também informava que, caso a criança colaborasse, emitindo os comportamentos ensinados (permanecendo com a boca aberta durante o exame clínico, por exemplo), ao final da sessão ela receberia um brinde (balão de ar). Na cadeira odontológica, a dentista realizava exame clínico, aplicação de um corante sobre os dentes da criança utilizando um pincel, para evidenciar as superfícies dentais que continham a placa bacteriana (matéria formada por bactérias e detritos alimentares, responsável pela cárie) e orientava a mãe sobre a técnica de higiene bucal domiciliar. Antes da realização de cada procedimento, a dentista mostrava o equipamento e os instrumentos que seriam utilizados e permitia que a criança os manipulasse. Em seguida, realizava-se o treino de escovação e a limpeza profissional dos dentes, utilizando equipamento odontológico. Terminados estes procedimentos, a criança recebia o brinde mesmo se não houvesse colaborado plenamente, permitindo a realização somente de alguns dos procedimentos planejados.

A partir da segunda sessão, a dentista mostrava à criança os brinquedos planejados para a atividade lúdica e estabelecia a seguinte contingência: caso ela colaborasse, poderia brincar no final da sessão e receber o brinde. Para que a criança entendesse esta contingência, a dentista utilizava o seguinte argumento: se ela ajudasse a dentista, ficando quieta e abrindo sua boca para a realização do tratamento, este seria feito o mais rápido possível e então sobraria tempo para a realização da atividade lúdica ao término da sessão.

Durante um período de tempo que durou em média 24 minutos, a dentista utilizava estratégias de manejo do comportamento, tais como a distração (conversando sobre os brinquedos que estavam sobre a mesa de atividade lúdica ou sobre algum assunto de interesse para a criança), a explicação (mostrando os equipamentos e instrumentos que seriam utilizados, permitindo que a criança manipulasse estes objetos e pedindo sua colaboração para o tratamento), o reforçamento específico (elogiando a criança quando esta emitia algum comportamento colaborador) e o estabelecimento de regras (lembrando a criança sobre a contingência) (Possobon, Moraes \& Caetano, 1998). Caso a criança não permitisse o tratamento, a dentista encerrava a sessão e explicava que não haveria atividade lúdica e entrega do brinde, pois não havia sobrado tempo devido à sua não - colaboração.
A dentista não utilizava nenhum tipo de restrição ou ajuda física para forçar a criança a permitir o tratamento. $\mathrm{O}$ tratamento era considerado realizado quando, com a colaboração espontânea da criança, fossem feitos os seguintes procedimentos: anestesia tópica (pomada anestésica), anestesia injetável, instalação de isolamento absoluto (lençol de borracha preso ao dente a ser tratado com auxílio de um grampo especial, que proporciona um campo seco para o trabalho do dentista), preparo cavitário utilizando alta e/ou baixa - rotação (remoção de cárie com o "motor") e restauração definitiva do dente.

Os comportamentos emitidos pelo paciente e pela dentista durante as sessões de atendimento foram observados e registrados posteriormente, utilizando as fitas de VT.

A observação e registro dos comportamentos dos pacientes foram realizados com base no Código HBCD, desenvolvido por Allard e Stokes (1980), utilizado também por Stokes \& Kennedy (1980), com algumas modificações. As categorias de comportamento observadas e registradas foram:

(1) Movimentos de corpo e/ou cabeça: qualquer movimento de corpo e/ou cabeça, manifestado durante a execução de um procedimento odontológico, que atrapalhasse a atuação do dentista, porém sem interromper o procedimento, concomitante ou não com choro;

(2) Choro ou reclamações: presença de choro, gemido ou reclamação sobre os procedimentos, registrado somente quando ocorria isoladamente.

(3) Fuga: quando a criança interrompia um procedimento que estava sendo realizado, por meio de movimentos bruscos de corpo e/ou cabeça, na presença ou não de choro;

(4) Esquiva: comportamentos que não permitiam o início da realização do procedimento. Exemplo: quando a criança não seguia instruções, recusando-se a deitar-se na cadeira ou a abrir a boca.

Após as sessões, a pesquisadora e uma assistente de pesquisa treinada assistiam aos VTs e faziam registros independentes dos comportamentos emitidos pela criança e das estratégias usadas pela dentista, a cada 15 segundos, utilizando uma ficha de observação. Posteriormente, era verificada a concordância entre os observadores, para conferir fidedignidade aos dados coletados (o nível de concordância obtido foi em média 99,06\%). Em seguida, foram calculadas as freqüências com que ocorreram os comportamentos, avaliando o grau de colaboração da criança ao longo das sessões.

\section{Resultados e Discussão}

O delineamento planejado para este estudo estabelecia que o tratamento fosse realizado somente se houvesse colaboração por parte da criança. Esta determinação permitiu que a criança tivesse a oportunidade de colaborar nas sessões em que recebeu placebo e naquelas em que recebeu diazepam. Além disso, possibilitou a observação sistemática dos comportamentos das crianças nas duas condições experimentais planejadas, utilizando-se estratégias comportamentais não - aversivas de controle do comportamento.

A Figura 1 apresenta as frequiências relativas dos comportamentos de não - colaboração que ocorreram em cada 
Grupo A

Grupo B
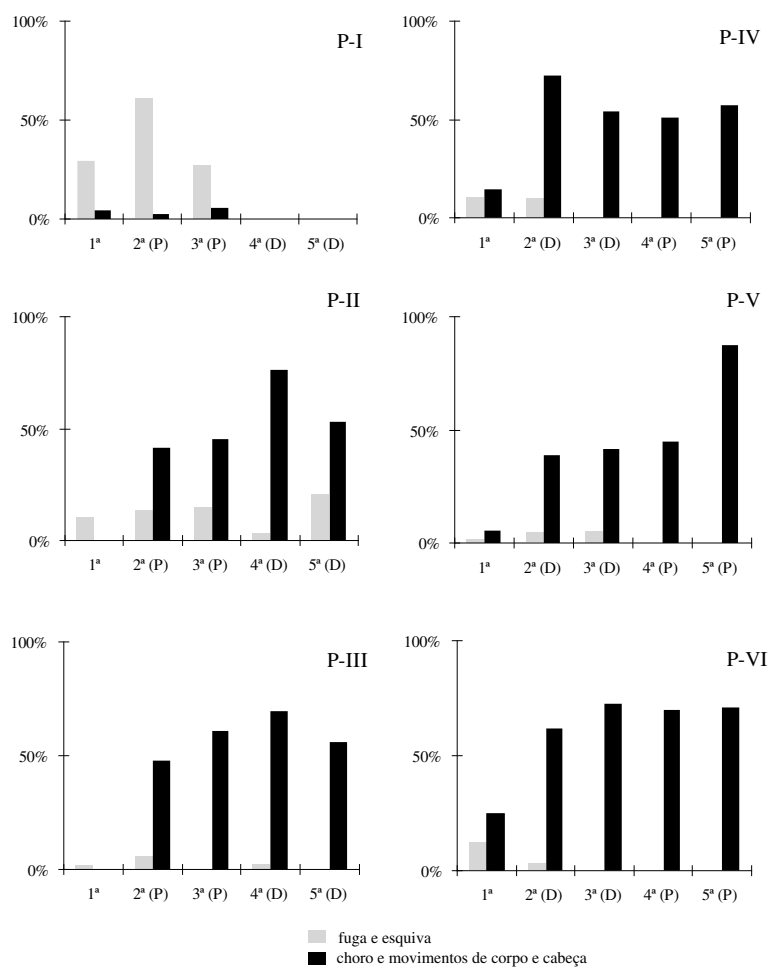

Figura 1. Freqüência relativa de comportamentos de não-colaboração de cada sujeito, por sessão.

sessão dos seis pacientes. Os comportamentos dos participantes foram agrupados desta maneira porque indicam os momentos em que a dentista está atuando (quando a criança chora ou movimenta-se) e os momentos em que a criança não permite a atuação da dentista (ocorrência de respostas de fuga e esquiva).

Observando-se as colunas preenchidas em preto (comportamentos de fuga ou esquiva), verifica-se um aumento gradual na frequiência relativa destes comportamentos, exceto no que diz respeito aos dados de P-I. Parece que o fato da criança perceber que o tratamento não seria realizado caso ela não permitisse, fortaleceu a emissão de comportamentos de esquiva, aumentando a não - colaboração ao longo das sessões. Além disso, houve também um aumento na intensidade destes comportamentos, ou seja, a criança parecia estar cada vez mais familiarizada, mas demonstrava comportamentos cada vez mais fortes, reagindo com agressividade ao contato da dentista. Este fato, impossível de ser visto pela observação desta figura, foi percebido pela pesquisadora e pela assistente de pesquisa e registrado sob a forma de relato cursivo ao final de cada sessão.

Vale a pena ressaltar que, exceto P-I, nenhum participante permitiu a realização de procedimentos da Segunda a quinta sessão. Portanto, a porcentagem de tempo das sessões em que não aparecem comportamentos de não - colaboração, refletem períodos em que a dentista estava realizando tarefas preparatórias para as intervenções, e não que a criança está colaborando e permitindo o tratamento.

A ocorrência do reforçamento requer a satisfação de dois critérios: o comportamento alvo precisa ser seguido por uma conseqüência que, por sua vez, aumenta a frequiência deste comportamento. O reforçamento pode ser usado pelo dentista de maneira deliberada ou ocorrer de forma não planejada (Moraes \& Pessotti, 1985). O brinquedo e o brinde foram estabelecidos como estímulos reforçadores na primeira sessão de cada participante. Todavia, a explicitação da não ocorrência do estímulo reforçador positivo, estabelecida na contingência, pode ter atuado como um estímulo discriminativo para não colaborar, uma vez que, não colaborando, a criança era dispensada e, desta maneira, negativamente reforçada. Assim, entre a primeira e a quinta sessão, os comportamentos de não - colaboração foram negativamente reforçados, ou seja, a criança não colaborava e o tratamento não era realizado. Allen e Stokes (1987) também concluíram que oferecer um prêmio contingente a comportamentos de colaboração durante o tratamento odontológico não foi suficiente para reduzir a não - colaboração.

As verbalizações (estratégias de distração, explicação e reforçamento), de maneira geral, foram bastante utilizadas. Tal como afirmaram Ten Berge, Veerkamp e Hoogstraten (1999), os comportamentos do dentista são dependentes do comportamento do paciente e, portanto, quanto mais alto for o nível de não - colaboração, maior a freqüência de utilização de estratégias de manejo do comportamento, empregadas para obter a colaboração. Neste sentido, pode-se supor que, em muitas circunstâncias, quem controla exatamente o transcorrer da sessão é o paciente e não o dentista. Como a distração e a explicação não podem ser realizadas ao mesmo tempo, quando a criança não está colaborando utiliza-se mais a explicação, como uma forma de convencer a criança a colaborar. Entretanto, parece que a explicação reforçou os comportamentos de esquiva, uma vez que deixava claro que, se a criança não colaborasse, a sessão seria encerrada e o tratamento não realizado.

Pinkham (1993) afirma que pedidos e promessas, mecanismos comumente utilizados para persuadir as pessoas, não produzem os efeitos esperados quando utilizados com pacientes indisciplinados. Estas crianças parecem ter aversão à autoridade do adulto e estão acostumadas a quebrar e ter suas promessas quebradas. Assim sendo, o dentista muitas vezes não consegue a colaboração destas crianças quando faz pedidos e promessas.

Ao final das sessões em que nenhuma intervenção havia sido feita, a dentista cumpria a regra estabelecida na contingência, ou seja, as crianças não poderiam brincar e nem receber o brinde. Nestas circunstâncias, os participantes saíam do consultório apresentando comportamentos que pareciam revelar raiva ou frustração. Mostravam-se, por vezes, agressivos com o acompanhante e choravam.

Os participantes deste estudo apresentavam história prévia de comportamentos de não - colaboração, fato que também pode explicar a ineficácia das estratégias utilizadas para manejar seus comportamentos. Os comportamentos apresentados pelas crianças na sessão de avaliação (antes de sua inclusão como participante deste estudo) e na sessão de linha de base (primeira sessão de atendimento), impediram a realização de procedimentos simples e não - invasivos, tais como o exame clínico, evidenciação de placa ou aplicação de flúor. Desta maneira, pode-se inferir que, se os participantes apresentassem menor grau de não - colaboração inicial, as estratégias comportamentais em associação com a medicação 
utilizada poderiam ter sido eficazes. Entretanto, esta é uma inferência prematura a partir dos dados disponíveis.

Observando ainda a Figura 1, verifica-se que para P-II, P-III (grupo A) e P-IV (grupo B), a colaboração foi menor, ou seja, houve uma maior freqüência na ocorrência dos comportamentos de fuga e esquiva (que impedem a atuação da dentista) nas sessões com diazepam (colunas com linhas oblíquas) comparadas às sessões com placebo. Com P-V e P-VI (grupo B), houve maior frequiência destes comportamentos nas sessões com placebo. Entretanto, principalmente para P-II e P-III (grupo A) e para P-V e P-VI (grupo B), houve uma tendência a aumentar os comportamentos de fuga e esquiva ao longo das sessões. Portanto, a quarta e quinta sessões foram aquelas em que houve uma frequiência maior de fuga e esquiva, seja com placebo ou diazepam. Por isso, não se pode afirmar que o diazepam tenha tido uma influência negativa nos comportamentos de P-II e P-III; apenas mostrou-se ineficaz na dose empregada.

Sabendo que nestas sessões, exceto para P-I, nenhuma criança permitiu a realização do tratamento, confirma-se que o diazepam não foi eficaz no controle dos comportamentos dos pacientes, nem tampouco as estratégias comportamentais. Embora colaborador, P-I não adormeceu e não estava sonolento nas sessões com diazepam.

Para ter sido considerada eficaz, a medicação não precisaria ter provocado sedação, levando a criança a dormir durante toda a sessão. O objetivo do emprego do ansiolítico era tranqüilizar a criança, deixando-a mais receptiva às estratégias de manejo empregadas, possibilitando a realização plena do tratamento, sem necessidade de contenção física.

\section{Considerações Finais}

O diazepam (0,3 mg/kg, p.o.) não se revelou eficaz para o controle dos comportamentos de não - colaboração.

Não houve mudanças no grau de colaboração dos participantes quando comparadas as sessões com medicação e com placebo, exceto para um participante, cujo nível de colaboração já era maior do que dos demais participantes.

A não realização do tratamento enquanto a criança não colaborou, reforçou as manifestações dos comportamentos de não - colaboração, aumentando sua freqüiência ao longo das sessões. A criança parece aprender que o comportamento de esquiva a livra da realização do tratamento. Parece importante auxiliar a criança a enfrentar a situação de atendimento odontológico assim que uma interação inicial seja estabelecida com o profissional ( $2^{\mathrm{a}}$ ou $3^{\mathrm{a}}$ sessão). Isto pode ser conseguido por meio da utilização de estratégias que restrinjam os movimentos da criança, associadas com procedimentos que induzam colaboração e possibilitem o enfrentamento dos eventos aversivos inerentes a situação odontológica.

\section{Referências}

Album, M.M. (1961). Meperidine And Promethazine Hydrochloride For Handicapped Pacients. Journal of Dentistry Research, 40,1036-1041.

Allard, G. \& Stokes, T.F. (1980). Continuous observation: a detailed record of children's behavior during dental treatment. Journal of Dentistry for Children, 47(4), 246-250.
Allen, K.D., Loiben, T., Allen, S.J. \& Stanley, R.T. (1992). Dentist - implemented contingent escape for management of disruptive child behavior. Journal of Applied Behavior Analysis, 25(3), 629-636.

Allen, K.D. \& Stokes, T.F. (1987). Use of escape and reward in the management of young children during dental treatment. Journal of Applied Behavior Analysis, 20(4), 381-390.

American Academy of Pediatric Dentistry. (1997-1998). Behaviour management manual Pediatric Dentistry, Special Issue: quality assurance criteria for Pediatric Dentistry - section III: 19(7), 90-91.

Badalaty, M.M., Houpt, M.I., Koenigsberg, S.R., Mawell, K.C. \& Desjardins, P.J. (1990). A comparison of chloral hidrate and diazepam sedation in young children. Pediatric Dentistry, 12(1), 33-37.

César, J. (1988). Caracterização comportamental do tratamento odontológico de pacientes especiais: estudo de dois casos. Dissertação de Mestrado. Universidade Federal de São Carlos, São Carlos, SP.

Costa, S.M. \& Moraes, A.B.A. (1994). Medo em Odontologia: um estudo com escolares. Revista Brasileira de Odontologia, 51(5), 26-31.

Healy. T.E.J. \& Hamilton, M.C. (1971). Intravenous Diazepam in the apprehensive child.British Dentistry Journal, 130(1), 25-27.

Ingersoll, B.D. (1982). Behavioral aspects in Dentistry (pp.107-33). N. York: Appleton Century - Crofts.

Lundin, R.W. (1972) Personalidade. Uma análise do comportamento. São Paulo: Herde.

Moraes, A.B.A. (1999) Comportamento e Saúde: explorando alternativas (pp.61-83). São Paulo: Arbytes.

Moraes, A.B.A. \& Pessotti, I. (1985) Psicologia aplicada à Odontologia. São Paulo: Sarvier.

Piedalue, R.J. \& Milnes, A. (1994). Nonpharmacological techniques help practitioners manage young patients. Journal of Massachusetts Dentistry Society 43(1), 247-251.

Pinkham, J.R. (1993). The roles of requests and promises in child patient management. Journal of Dentistry for Children, 60(3), 169-174.

Possobon, R.F., Caetano, M.E.S., Moraes, A.B.A. (1998). Odontologia para crianças não - colaboradoras: relato de casos. Revista Brasileira de Odontologia, 55(2), 80-83.

Stark, L.J., Allen, K.D., Hurst, M., Nash, D.A., Rigney, B. \& Stokes, T.F. (1989). Distraction: its utilization and efficacy with children undergoing dental treatment. Journal of Applied Behavior Analysis, 22(3), 297-307.

Sams; D.R., Cook, E.W., Jackson, J.G. \& Roebuck, B.L. (1993). Behavioral assessment of two drug combinations for oral sedation. Pediatric Dentistry, 15(3), 186-190.

Stokes, T.F. \& Kennedy, S.H. (1980). Reducing child uncooperative behavior during dental treatment through modeling and reinforcement. Journal of Applied Behavior Analysis, 13(1), 41-49.

Ten Berge, M., Veerkamp, J. \& Hoogstraten, J. (1999). Dentists' behavior in response to child dental fear. Journal of Dentistry for Children,66(1), 36-40.

Wolpe, J. (1971). The Conditioning and Deconditioning of Neurotic Anxiety. Em C.D. Spielberger (Org.), Anxiety and Behavior (pp. 179-190). N. York: Academic Press.

Yanase, H., Braham, O.F., Fukuta, O. \& Kurosu, K. (1996). A study of the sedative effect of home-administered oral diazepam for 
R. F. Possobon \& cols.

the dental treatment of children. International Journal of Paediatric Dentistry, 6(1), 13-17.
Recebido em 09.04.2002

Primeira decisão editorial em 16.08.2002

Versão final em 05.04.2003

Aceito em 15.04.2003 